\title{
Anne Quéniart
}

Sociologue, professeure, département de sociologie, UQÀM

(2004)

\section{"Regards de jeunes pères sur la famille et la paternité"}

\author{
Un document produit en version numérique par Jean-Marie Tremblay, bénévole, \\ professeur de sociologie au Cégep de Chicoutimi \\ Courriel: jean-marie tremblay@uqac.ca \\ Site web pédagogique : http://www.uqac.ca/jmt-sociologue/ \\ Dans le cadre de: "Les classiques des sciences sociales" \\ Une bibliothèque numérique fondée et dirigée par Jean-Marie Tremblay, \\ professeur de sociologie au Cégep de Chicoutimi \\ Site web: http://classiques.uqac.ca/ \\ Une collection développée en collaboration avec la Bibliothèque \\ Paul-Émile-Boulet de l'Université du Québec à Chicoutimi \\ Site web: http://bibliotheque.uqac.ca/
}




\section{Politique d'utilisation de la bibliothèque des Classiques}

Toute reproduction et rediffusion de nos fichiers est interdite, même avec la mention de leur provenance, sans l'autorisation formelle, écrite, du fondateur des Classiques des sciences sociales, Jean-Marie Tremblay, sociologue.

Les fichiers des Classiques des sciences sociales ne peuvent sans autorisation formelle:

- être hébergés (en fichier ou page web, en totalité ou en partie) sur un serveur autre que celui des Classiques.

- servir de base de travail à un autre fichier modifié ensuite par tout autre moyen (couleur, police, mise en page, extraits, support, etc...),

Les fichiers (.html, .doc, .pdf., .rtf, .jpg, .gif) disponibles sur le site Les Classiques des sciences sociales sont la propriété des Classiques des sciences sociales, un organisme à but non lucratif composé exclusivement de bénévoles.

Ils sont disponibles pour une utilisation intellectuelle et personnelle et, en aucun cas, commerciale. Toute utilisation à des fins commerciales des fichiers sur ce site est strictement interdite et toute rediffusion est également strictement interdite.

L'accès à notre travail est libre et gratuit à tous les utilisateurs. C'est notre mission.

Jean-Marie Tremblay, sociologue

Fondateur et Président-directeur général, LES CLASSIQUES DES SCIENCES SOCIALES. 
Cette édition électronique a été réalisée par Jean-Marie Tremblay, bénévole, professeur de sociologie au Cégep de Chicoutimi à partir de :

\section{Anne Quéniart}

Sociologue, professeure, département de sociologie, UQÀM

“Regards de jeunes pères sur la famille et la paternité".

Un article publié dans l'ouvrage sous la direction de Gilles Pronovost et Chantal Royer, Les valeurs des jeunes, chapitre 6, pp. 111-130. Québec : Les Presses de l’Université du Québec, 2004, 252 pp.

[Autorisation formelle accordée par Mme Quéniart le 7 août 2008 de diffuser cet article dans Les Classiques des sciences sociales.]

Courriels : Anne Quéniart : queniart.anne@uqam.ca

Polices de caractères utilisée :

Pour le texte: Times New Roman, 12 points.

Pour les citations : Times New Roman, 12 points.

Pour les notes de bas de page : Times New Roman, 12 points.

Édition électronique réalisée avec le traitement de textes Microsoft Word 2004 pour Macintosh.

Mise en page sur papier format : LETTRE (US letter), 8.5’’ x 11'’)

Édition numérique réalisée le 8 juillet 2008 et revue le 16 juin 2010 à Chicoutimi, Ville de Saguenay, province de Québec, Canada.

\section{CFait avec}




\section{Anne Quéniart}

Sociologue, professeure, département de sociologie, UQÀM

\section{"Regards de jeunes pères} sur la famille et la paternité.”

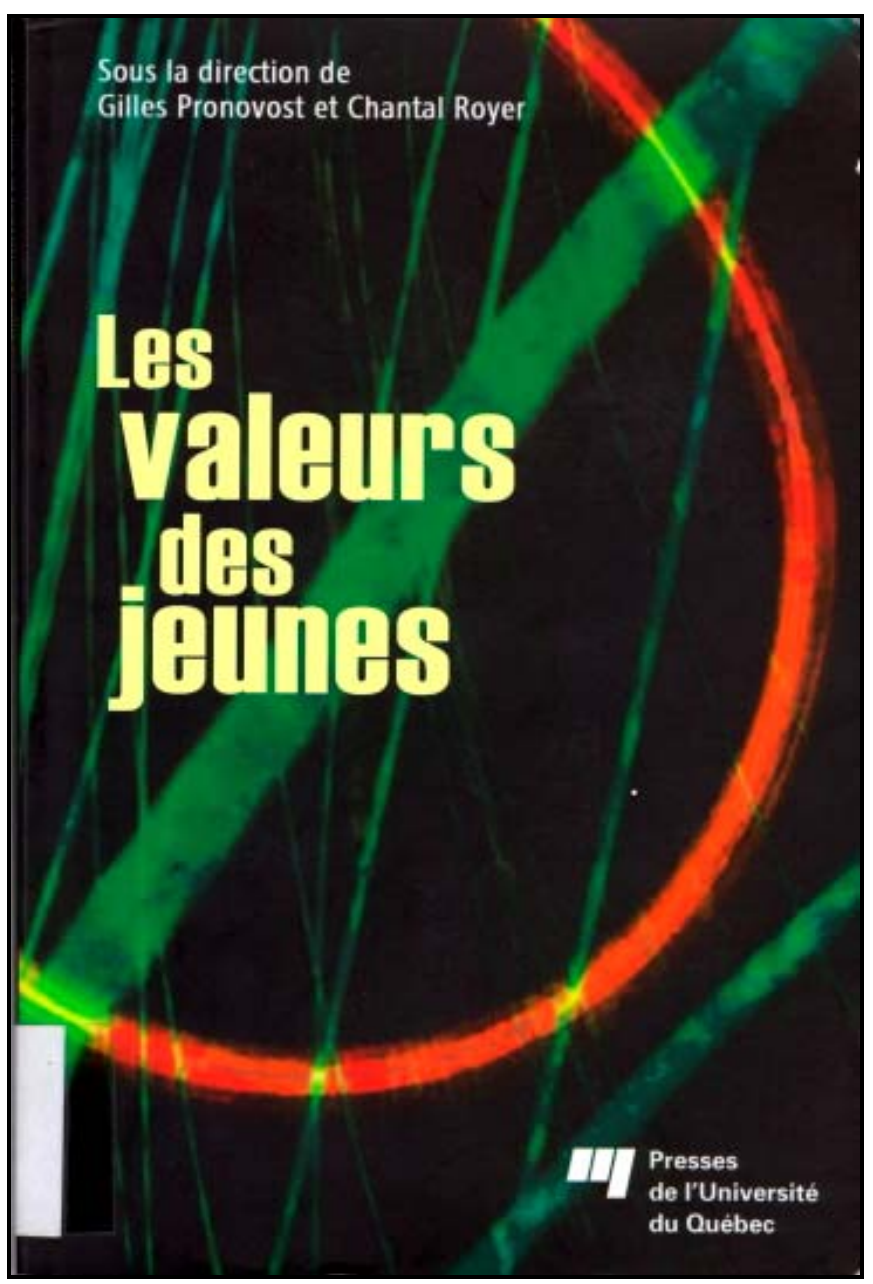

Un article publié dans l'ouvrage sous la direction de Gilles Pronovost et Chantal Royer, Les valeurs des jeunes, chapitre 6, pp. 111-130. Québec : Les Presses de l’Université du Québec, 2004, 252 pp. 


\section{Table des matières}

$\underline{\text { Introduction }}$

Que sait-on des valeurs accordées par les pères à la famille et à la paternité? Aspects méthodologiques de la recherche

Description de l'échantillon

Réalisation et analyse des entrevues

Les valeurs qui définissent la famille

La famille: un arbre à trois branches

Une centration sur l'enfant

L'autonomie : une valeur primordiale

Une prépondérance des valeurs de la famille sur celles de l'extérieur

Les valeurs privilégiées dans la définition de la paternité

Un rôle paternel basé sur la présence

$\underline{\text { Une relation père/enfant sous le signe de la démocratie familiale }}$

$\underline{\text { Conclusion }}$

Bibliographie 


\section{Anne Quéniart \\ Sociologue, professeure, département de sociologie, UQÀM \\ “Regards de jeunes pères sur la famille et la paternité”. 1}

Un article publié dans l'ouvrage sous la direction de Gilles Pronovost et Chantal Royer, Les valeurs des jeunes, chapitre 6, pp. 111-130. Québec : Les Presses de l’Université du Québec, 2004, 252 pp.

\section{Introduction}

$\underline{\text { Retour à la table des matières }}$

La famille, cela signifie quoi pour des jeunes hommes qui sont devenus pères à un âge où la plupart d'entre eux étudient, commencent leur vie professionnelle ou encore voyagent? Quelles valeurs privilégient-ils dans la définition de leur rôle de parent? Dans quelles pratiques de paternité ces valeurs se traduisent-elles? Quelles représentations de l'enfant sont associées à ces valeurs? Telles sont quelques-unes des questions auxquelles je me propose de répondre dans ce texte à partir des données d'une recherche qualitative par entrevues en profondeur menée à Montréal auprès de plus d'une trentaine de jeunes devenus pères entre l'âge de 17 et 24 ans $^{2}$. Bien que centrée plutôt sur l'expérience de la paternité au quotidien, cette recherche met en lumière un certain nombre de valeurs auxquelles les jeunes pères se réfèrent lorsqu'ils décrivent leurs pratiques, leurs façons d’être et de faire avec leur enfant. En effet, comme Guy Rocher le souligne bien, la valeur possède ce caractère particulier d'être inscrite à la fois dans l'ordre de l'idéal et dans la réalité :

1 Ce texte reprend des éléments d'analyse déjà présentés ailleurs (Quéniart 2002, 2003).

2 Recherche financée par le Conseil québécois de recherche sociale. 
«En tant qu’idéal, la valeur implique donc l’idée d’une qualité d’être ou d'agir supérieure à laquelle on aspire ou dont on s’inspire. À ce titre cependant, la valeur n'est pas moins réelle que les conduites ou les objets dans lesquels elle se concrétise ou par lesquels elle s'exprime» (Rocher $1969: 56)$

Nous tenterons donc ici de dégager comment se présente la famille pour ces jeunes pères et de mettre au jour les valeurs qu'ils privilégient, qu'ils sélectionnent en priorité, dans la description de leurs pratiques paternelles au quotidien.

\section{Que sait-on des valeurs accordées par les pères à la famille et à la paternité?}

$\underline{\text { Retour à la table des matières }}$

Peu de recherches sociologiques, au Québec comme ailleurs, se sont intéressés aux jeunes pères, c'est-à-dire à cette catégorie charnière entre l'adolescence et l'âge adulte qu'est celle des 18-25 ans. En outre, peu de recherches se sont penchées sur la question des valeurs accordées à la famille ou à la parentalité ${ }^{3}$ ou plutôt l'ont fait mais indirectement, notamment par le biais de typologies des rôles parentaux ou familiaux. En effet, les typologies sont utilisées abondamment en sociologie, et particulièrement dans le champ de la famille (Ménahem 1979, Kellerhas 1987, Russel 1983). Leur utilité en est une surtout de «classement des objets sociaux, [de] conceptualisation d’indicateurs agrégés ou plus encore [de] prédiction de comportements probables en telle ou telle matière» (Bawin-Legros et Sommer 1987: 47). Dans la littérature scientifique consacrée spécifiquement à la paternité, on rencontre différents genres de typologies, certaines se centrant plutôt sur les pratiques paternelles (Broderick 1977, Robinson et Barret 1986, Palm et Palkovitz 1988), d’autres sur les conceptions du rôle paternel et de la paternité

3 En ce qui a trait à la paternité, de nombreuses recherches, notamment dans le champ de la psychologie, portent sur la question des déterminants de l'implication paternelle. Voir pour une synthèse à ce sujet Turcotte (1994). Pour ce qui est des valeurs des jeunes, les enquêtes se centrent plutôt sur les adolescents et abordent surtout les valeurs liées au domaine général de la culture (musique, mode, loisir, religion, etc.). Pour le Québec, voir Gauthier (1997), Kerr et al. (1994) et Secrétariat à la jeunesse (1992). 
(Ferrand 1981, Pleck 1987, Lamb 1987, Dycke et Saucier 1999; Dienhart 1998, Delaisi de Parseval 1982, Quéniart 2002a, Fournier et Quéniart 1994). Tous les auteurs font d'abord le constat d'une pluralité de pratiques et de valeurs liées à la paternité, celles-ci variant selon les contextes sociaux (Erickson et Gecas 1991, Arama et Bouchard 1996, Lévesque, Perrault, Goulet 1997, Dienhart 1998, Quéniart 2002a) et selon les cultures (Lamb 1987, Iishii-Kuntz 1994, Dycke et Saucier 1999).

Au sein des divers modèles qui coexistent aujourd'hui, les chercheurs s'entendent pour dire que l'on peut dégager trois types-idéaux de paternité. Le premier type renvoie au père traditionnel, appelé aussi père pourvoyeur (Fournier et Quéniart 1994, Lévesque, Perrault, Goulet 1997, Quéniart 2002a), père autocrate ou patriarche (Broderick 1977) dont le rôle est ramené essentiellement à la dimension économique et à celle de l'autorité et dont le rapport à l'enfant est médiatisé par la mère; le second type est représenté par le nouveau père (Delaisi de Parseval1982, Dienhart 1998) parfois nommé aussi père androgyne (Rotundo 1985, Robinson et Barret 1986, Verheyen 1987), co-parent (Russel 1982, Palm et Palkovitz 1988), leader expressif (Broderick 1977) ou encore père substitutif (Ferrand 1981) dont le rôle comporte des dimensions à la fois relationnelles, éducatives et de responsabilité matérielles, dont la relation à l'enfant est personnelle et directe et basée sur l'expression de l'affectivité; un troisième type émerge de certaines études, le père assistant-parent (Russel 1982, Palm et Palkovitz 1988) dit aussi père complémentaire (Ferrand 1981), ou père typique (Robinson et Barret 1986) ou encore père téflon (Fournier et Quéniart 1994) dont le rôle emprunte certains éléments du nouveau-père (relation personnelle à l'enfant) mais aussi au père traditionnel (médiatisation de la mère, importance du rôle de pourvoyeur).

La plupart des auteurs soulignent que les choses ont changé depuis quelques décennies et que le modèle du père traditionnel est de plus en plus concurrencé par celui du nouveau père. Fait étonnant cependant, dans ces typologies, on ne fait pas d'analyse selon la catégorie d’âge. Pourtant, si des changements se sont produits, on devrait les constater chez les plus jeunes parents. C'est en tout cas l'hypothèse qui sous-tend la recherche que j'ai menée auprès de jeunes pères. Ces jeunes ont en effet ceci de particulier qu'ils sont parmi les premiers à avoir été marqués, comme enfants, par les mutations qui ont secoué la famille au Québec, 
qu'il s'agisse de l'augmentation fulgurante des taux de divorce, de la montée de l'union libre ou encore de la baisse de la nuptialité (Dandurand 1995). Nés entre les années 1976 et 1984, les jeunes que nous avons rencontrés font partie de cette génération d'enfants nés dans une famille restreinte -il sont souvent enfants uniques-, dont les deux parents ont été actifs -et le sont encore- et dont plus d'un sur deux a vu ses parents se séparer (17 jeunes sur 32); ils font aussi partie aussi de cette génération pour qui la famille et la paternité sont à construire, ne pouvant s'appuyer sur une définition aux contours clairs, contrairement aux hommes des générations précédentes (Delumeau et Roche 1990).

\section{Aspects méthodologiques de la recherche}

\section{Description de l'échantillon}

$\underline{\text { Retour à la table des matières }}$

Sur le plan méthodologique, nous avons opté pour l'approche qualitative de la théorisation ancrée (Paillé 1994, Laperrière 1998) qui est appropriée dans le cas de phénomènes ou de groupes sociaux peu étudiés. Des entrevues en profondeur ont été menées auprès de 32 jeunes pères, âgés de 19 à 26 ans au moment de la recherche, et qui ont eu leur premier enfant entre 17 et 24 ans, la moyenne étant de 21.4 ans. Trois des pères sont séparés de la mère de l'enfant; un autre père n'a jamais été en union conjugale avec la mère de son enfant. Les autres vivent en couple dont 8 étant mariés et 20 vivant en union libre. Quinze pères ont un diplôme d'études secondaire ou une scolarité moindre (secondaire 4), sept ont un diplôme d'études collégiale ou d'études professionnelle, sept un baccalauréat, un a un certificat universitaire et deux poursuivent actuellement leur scolarité de maîtrise. Ils ont des revenus personnels variant moins de 12, 000 à plus de 40,000 dollars, 12 d'entre eux gagnant 15, 000 et moins, quatre d'entre eux gagnant plus de 30000 dollars, les 16 autres ayant un revenu entre 15, 000 et 30, 000 dollars. Sur le plan de l'occupation, 13 jeunes pères travaillent à temps plein, trois à temps partiel, deux sont prestataires de l'assurance emploi lors de l'entrevue, neuf travaillent et étudient en même temps, parfois à temps plein, parfois à temps partiel et enfin, 5 étudient à temps plein. Deux des pères interviewés ont deux enfants, 5 
autres sont pères d'un enfant et en attendent un second. Deux hommes sont membres d'une famille recomposée autour de la mère avec un enfant en provenance d'une union antérieure : un des deux a eu un enfant, portant à deux les enfants à sa charge. Les vingt-trois autres pères ont un seul enfant.

\section{Réalisation et analyse des entrevues}

$\underline{\text { Retour à la table des matières }}$

Lors des entrevues, d'une durée moyenne de 90 minutes, les pères étaient invités à nous «raconter leur expérience de père» et nous laissions émerger les thèmes qu’ils jugeaient importants à la compréhension de leur vécu, conformément aux principes de la théorisation ancrée. Cependant, nous avons veillé à ce que certains thèmes soient abordés systématiquement par tous les répondants, pour fins de comparaison, soit : le contexte de la venue de l'enfant, le rapport des jeunes pères à leur enfant et à la famille (représentations, pratiques quotidiennes), la place et le sens de la paternité en regard de leur vie personnelle et de leur vie de couple, de leur vie professionnelle, de leur vie sociale. Toutes les entrevues ont été enregistrées puis retranscrites intégralement et soumises à une analyse qualitative de contenu comportant deux niveaux. Le premier, celui de l'analyse verticale (contenu d'une entrevue) a comporté trois étapes. Nous avons d'abord repéré et codé tous les thèmes prévus dans le guide ou qui ont émergé lors des entrevues (contexte de la grossesse, réactions de l'entourage, activités avec l'enfant, etc.). Ensuite, nous avons effectué des regroupements en catégories («l’importance de la présence», «l'enfant comme prolongement de soi» etc.). Enfin nous élaborions des hypothèses visant à interpréter le discours des jeunes pères. Le second niveau d'analyse visait à comparer les contenus des discours des jeunes pères selon les variables indépendantes pertinentes (situation conjugale, occupation, etc.) et à raffiner les catégories créées. On constatera à cet égard peu de différences entre les pères quant à leurs représentations de leur rôle. En revanche, lors des analyses, 
des différences sont apparues sur la question du partage des tâches au sein de la famille ${ }^{4}$.

Dans ce texte, je m'attarderai dans un premier temps aux valeurs privilégiées par les jeunes pères dans la définition qu'ils donnent de la famille et, dans un second temps, à celles qu'ils considèrent comme prioritaires, essentielles à l'exercice de leur rôle de père au quotidien.

\section{Les valeurs qui définissent la famille}

$\underline{\text { Retour à la table des matières }}$

Il est toujours réducteur de vouloir ramener à quelques dimensions des représentations de phénomènes ou d'expériences complexes comme la famille ou la paternité. En même temps, c'est un exercice qui permet de jeter un regard d'ensemble sur l'«état» de la famille d'aujourd'hui, telle que vue par ceux qui en sont des acteurs privilégiés, soit les pères. Dans les récits de ceux que nous avons rencontrés, quatre aspects sont ressortis comme étant incontournables à leur appréhension de la famille, à savoir : l'importance à la fois de la «stabilité du tout» et de «l'interdépendance des parties», la centration sur l'enfant, l'autonomie par rapport à la parenté, la prépondérance des valeurs familiales sur celles de l'extérieur.

\section{La famille : un arbre à trois branches}

L'image qui vient d'abord à l'esprit lorsque l'on se penche sur les descriptions faites par les pères de ce qu'est pour eux la famille, est celle d'un arbre à trois branches, celles-ci représentant la mère, le père et l'enfant. Ce dernier est défini comme essentiel à la définition même de la famille : «quand t'as un enfant, tu deviens une famille, tu n'es plus un couple». Pour ces jeunes nés à la fin des années 1970 et au début des années 1980, la formation du couple n’est plus, comme

4 Le manque d'espace nous empêche de développer ici cette question. Voir pour plus de détails Quéniart (sous presse). 
dans les décennies précédentes, l'acte de fondation de la famille. C’est plutôt la venue de l'enfant qui marque véritablement l'entrée dans l’âge adulte (Dandurand 1995).

L’image de l'arbre renvoie d'abord à l'une des valeurs considérées comme fondamentale par les jeunes, soit celle de la stabilité du tout qu'est la famille : celle-ci est le socle sur lequel l'enfant peut se reposer, son point d'ancrage dans la vie, ses racines, et il importe que ce pilier reste fort, en l'occurrence que le couple «reste uni pour la vie» :

Une famille idéale c’est évidemment une famille qui est ensemble, une famille unie puis une famille qui est stable, qui reste, sans avoir de routine, être capable de rester ensemble, tous au même endroit, assez longtemps pour pas que l'enfant soit désorienté ça c’est assez important. (Gabriel, 21 ans, DEP, un enfant d'un an).

Ben je dirais, idéalement j’aimerais ça resté avec ma blonde, qu’on soit un couple uni, qu'on ne se sépare pas (Adam, 25 ans, un enfant d'un an, baccalauréat).

C'est que la famille reste unie parce que je suis à peu près le seul dans mon entourage qui a encore ses deux parents, ensemble. Ça je trouve que, je ne sais pas si moi pis ma blonde on va faire notre vie ensemble, mais j'aimerais ça. J'aimerais ça pour la petite, j'aimerais ça pour moi aussi évidemment, pour nous deux mais surtout pour la petite. Moi étant donné que je suis à peu près le seul qui a gardé ses parents, c'est quasiment rendu que tu te sens différent parce que tu as tes deux parents, parce qu'ils ne sont pas séparés. (Alexis, 19 ans, un enfant de 8 mois, secondaire 4).

Par ailleurs, les trois branches de l'arbre symbolisent également les trois types de relations qui décrivent la famille chez les jeunes pères c'est-à-dire la relation filiale, dont nous reparlerons dans la seconde section de ce texte, la relation conjugale et la relation parentale qui ont chacune leur dynamique propre, qui sont interdépendantes. Pour eux en effet, une famille c'est un ensemble de relations interpersonnelles indépendantes bien que liées les unes aux autres. On ne retrouve pas chez eux cette idée de la famille formant un tout indissociable, que l'on retrouve chez plusieurs pères des générations précédentes (Fournier et Quéniart 1994, Dienhart 1998); au «Nous familial» des hommes des générations précédentes -les «Pères de famille»-, on substitue ici les multiples «Je» interreliés, soit leur 
propre «je» de parent, de conjoint et d’homme, celui de leur conjointe également femme et parent, et celui de l'enfant. Chacun des membres de la famille a ainsi plusieurs identités, et les jeunes pères se présentent à la fois comme des jeunes hommes, des parents, des pères, des amoureux. Ces identités et les divers rôles qui leur sont associés ne sont d'ailleurs pas toujours faciles à concilier. Ainsi, lorsqu'ils parlent de leur nouvelle vie familiale, les jeunes pères distinguent pour la plupart nettement le conjugal du parental. Idéalement, disent-ils, le conjugal doit rester le lieu distinct, non routinier, de la vie amoureuse. On observe un désir de préserver l'autonomie de la relation de couple face à l'absorption dans la routine parentale en trouvant un moment de la journée consacrée exclusivement au couple (par exemple, après avoir couché l'enfant) ou en se ménageant des sorties de couple :

Avant que ma fille se couche je ne la vois pas ma blonde, c'est ma vie de famille. Un coup que ma fille est couchée, bien là je vois ma blonde. Tu sais c'est d'autre chose avant ce n'était pas pareil, avant c'était juste ma blonde. (Mathieu, 24 ans, baccalauréat, un enfant de trois ans)

Il y a des chicanes puis ma femme me dit faut que tu aies plus de temps pour le couple. Et moi je me dis que si j’ai assez aimé ma blonde pour lui faire un enfant, il y a sûrement moyen que je fasse quelque chose pour l'aimer encore. Alors, faire garder la petite, c'est comme entretenir le feu de notre couple! (Étienne, 24 ans, un enfant de 2 ans, baccalauréat).

La relation parentale, quant à elle, est décrite en termes d'ententes et de partage entre les deux parents, chacun gardant néanmoins sa spécificité comme parent, dans sa relation à l'enfant. Plusieurs jeunes soulignent à cet égard, qu'un père ce n’est pas une mère et qu’il faut respecter les goûts et les désirs de chacun.

\section{Une centration sur l'enfant}

$\underline{\text { Retour à la table des matières }}$

L'enfant occupe une place à part dans cet arbre que représente la famille puisqu'il en est non seulement le «ferment», mais aussi le «ciment». Il est le prisme à partir duquel les jeunes pères organisent dorénavant leur quotidien. Ils en parlent 
tous en disant «mon fils» ou «ma fille», non pas au sens d'une quelconque exclusion de la mère, mais bien plutôt en vertu du type de relation que ce père entretient avec son enfant, à savoir une relation personnelle et directe, proche et intense, comme nous le verrons plus loin. La centration sur l'enfant s'exprime de multiples façons et tout d'abord par le fait que sa venue vient bousculer et transformer la vie quotidienne du jeune père. En effet, plusieurs jeunes, à l'annonce de la venue de l'enfant (non prévue dans trois quarts des cas), vont être amenés à faire des changements de vie, notamment de carrière :

Avant qu'elle me dise qu'elle était enceinte, j'avais commencé un DEC de trois ans en théâtre mais c'est ça, étant que le théâtre c'est rien de garanti comme revenu, moi, j'aurai vécu de ça mais il faut que tu penses au travail plus tard. Je me suis trouvé quelque chose d'autre, que j'aimais aussi pis que c'est ben payant, pour tout de suite je travaille dans le plastique pis je me suis inscrit pour l'automne en technologie de la maintenance industrielle. (Charles, 19 ans, un enfant d'un an, DES, travailleur dans une usine de plastique)

Disons que ça a tout remis en question. On voit toujours sa vie d'une certaine façon et quand il y a un enfant qui arrive, ça défait un peu les plans. On pensait partir deux fois en voyage, on pensait rester dans un petit appartement, pis aussi faire nos études plus rapidement, en finir rapidement avec la maîtrise. (...) A partir du moment où on a appris ça, on s'est mis aux études à temps partiel parce qu'il fallait qu'on travaille beaucoup plus pour ramasser de l'argent. (Adam 25 ans, un enfant d'un an, baccalauréat, assistant de recherche à l'université et étudiant).

Les six premiers mois, je m'en suis pas occupé beaucoup je veux dire je m'en occupais le soir, je travaillais comme soixante dix heures par semaine, j'étais dans la construction. C'est difficile, donc j'ai arrêté puis j'ai fait un cours d'agent d'immeuble. C'est payant, puis c'est valorisant comme travail pis les horaires sont flexibles. Justement, pour aller chez le médecin l'après-midi, ben ça je peux y aller, je peux toujours me libérer. Il n’y a personne de plus disponible que moi, tu comprends-tu? (William 22 ans, un enfant de 3 ans, DEP).

Tous ces réaménagements témoignent certes du sens des responsabilités de ces jeunes mais aussi de la place qu'ils tiennent à occuper auprès l'enfant et de leur implication concrète et quotidienne. 
Un autre indice de la centration sur l'enfant, est la priorité des jeunes pères qui va plutôt à l'enfant qu’à la vie sociale.

Depuis qu'elle est née, j’ai fini mon secondaire, je suis rendu au CEGEP, puis mes amis je les vois un petit moins mais ça ne me dérange pas. Passer une soirée avec ma fille, j'aime mieux ça que de boire une bière. Tout ça, genre côté amis, école, ça ma réveillé. (Francis, 21 ans, diplôme d'études secondaires, un enfant d'un an).

Il est intéressant de constater que l'attrait de la sphère privée participe chez plusieurs jeunes hommes à la réévaluation des désirs de sortie. Une fois le deuil de la vie de jeunesse réalisé, on semble développer un réel attachement pour une vie plus casanière et plus tranquille «en famille», «à la maison» :

Au lieu d'avoir une vie sociale dans des lieux publics, on a une vie sociale dans un milieu privé, on va chez du monde. (...) Je ne peux pas suivre (mes amis) tout le temps puis tu développes à un moment donné un attachement assez grand envers ta vie familiale (Mathieu, 24 ans, baccalauréat, un enfant de trois ans)

On fait plus d'affaire chez nous. On ne sort plus vraiment dans les bars. On invite beaucoup de monde chez nous. C'est plus là que ça se passe, on reçoit beaucoup. (...) Avec un enfant, tu changes d'activités, tu vas faire quelque chose que tu peux faire avec ton bébé. C’est plus pépère, c'est sûr à un certain point de vue (Adam, 25 ans, baccalauréat, un enfant de 10 mois).

Enfin, un autre indice de la force du lien paternel à l'enfant ressort chez ceux qui ont vécu une séparation conjugale : ils ont revendiqué leur présence, leur implication, notamment en «négociant» une garde partagée de l'enfant :

Ça a été difficile de m’avouer vaincu par rapport au fait qu’on n’arrivera pas à faire cette belle petite famille unie qu'on voulait. Je veux vraiment être un bon père quand même et les voir le plus souvent possible. J'ai une grande partie dans leur éducation et j'ai vraiment l'intention que mes filles me perçoivent comme le modèle masculin. Je ne veux pas être le père manquant. Je veux vraiment être présent. (Laurent, 21 ans, un enfant d'un an et un à venir, séparé, DES) 


\section{L'autonomie : une valeur primordiale}

$\underline{\text { Retour à la table des matières }}$

Chez la plupart des jeunes pères interrogés, on note, dès l'annonce de la grossesse, une revendication très forte à l'autonomie paternelle et parentale et, corrélativement, une fermeture de «la nouvelle famille» à toute intrusion extérieure, en l'occurrence la parenté. Paradoxalement pourtant, les familles d'origine constituent le principal faisceau de soutien pour l'entrée en parentalité de ces jeunes et ce, sous des formes diverses : prêts ou dons monétaires, collecte de matériels pour l'arrivée du bébé, assistance soutenue pour le gardiennage. En fait, bien que pour la majorité des répondants la décohabitation d'avec leurs parents ait déjà été signée avant la venue du premier enfant (25 des 32 pères rencontrés), il n’y a pas encore une parfaite indépendance à l'égard de la famille d'origine. Cependant, pour la plupart de ces jeunes pères, cette relative dépendance ne doit pas empêcher l'établissement d'une frontière entre leur nouvelle entité familiale et leur famille d'origine, c'est-à-dire l'autonomie de leur propre système familial. Bref, si cette proche parenté «est bien un vecteur de soutien et de services, l'autonomie des membres de la famille doit demeurer la règle» (Martin : 2000, p. 109). Pour certains, cette étape peut également représenter le moment pour faire valoir devant son premier cercle de socialisation son passage définitif dans la « cour des adultes » :

Concrètement, je me suis mis à travailler plus. J'aurai pu ne pas travailler plus puis dire à mon père aide moi. Il me l'a offert, quand je lui ai annoncé que j'allais avoir un enfant, il a dit financièrement je vais t'aider. On ne l'a pas fait. Moi je me dit, tu veux l'avoir, bien faut que tu prouves que tu es capable, sinon ils vont toujours dire que ça a été une erreur puis que tu étais ben trop jeune. Tandis que si tu montres tout de suite que tu es capable puis que tu n'as besoin de personne, ils vont dire finalement qu'il ait 19 ans au lieu de 25, ça va bien (William, 22 ans, diplôme d'études professionnelles, un enfant de 3 ans).

Dans tout les cas, la recherche d'autonomie et ce, malgré la persistance d'un lien solide de dépendance, est apparue comme une revendication importante. Pour 
certains pères, un compromis avec la famille d’origine a pu aisément être mis en place et l'aide occasionnelle ou les conseils furent même appréciés. Pour d’autres, la recherche d'autonomie est devenue synonyme de vives tensions. Le support reçu de la part du cercle de parenté n’autorise aucunement une ingérence de celuici dans la façon dont ces jeunes pères élèvent leur enfant :

C’est pas sa mère à cet enfant là c'est sa grand-mère, c'est ma mère à moi. Arrête de me donner ton opinion sur tout, je ne la veux pas (...). Avec ma mère y'a une espèce d'ingérence dans ta vie privée, tu ne peux pas t'en défaire. À un moment donné ça devient tannant, c'est bien beau dire je veux t'aider, je veux te supporter, supporter mais laisses-moi faire mes choix quand même (Mathieu, 24 ans, baccalauréat, un enfant de trois ans).

Ma mère c'est du genre qui va s’ingérer dans ta vie, fait que quand elle a su qu'on avait un enfant, ben là, il fallait se marier au plus vite, puis là, elle avait quasiment réservé la salle de mariage. Fait que, c’était comme, oh, relaxe, prend une distance, en tout cas, on l'a comme tassée, on a organisé notre mariage nous-mêmes, et là, on s’est abonné au sélecteur de message sinon, elle appellerait à tous les jours pour nous dire quoi faire avec le petit! (Justin, 25 ans, baccalauréat, un enfant de 2 ans et un à venir).

Autrement dit, il s'agit de faire connaître ses limites à cet interventionnisme plein des meilleures intentions : le motus vivendi établissant la distance à respecter est alors établi par la nouvelle entité familiale, soucieuse de sa souveraineté, si virtuelle soit-elle. Si cette requête n’obtient pas l'écho escompté, l’attitude de la famille d'origine est ressentie comme de l'ingérence et devient totalement illégitime ; le fossé peut alors se creuser davantage, rendant l'accès à l'enfant encore plus protégé. Les notions de liberté et de choix nous sont apparues comme centrales pour la plupart des répondants. Certains préfèreront même limiter l'assistance en provenance du réseau familial afin de préserver leurs pouvoirs au sein du système familial balbutiant. La mise en place de l'autonomie passe donc parfois par l’indépendance matérielle elle-même... 


\section{Une prépondérance des valeurs}

de la famille sur celles de l'extérieur

$\underline{\text { Retour à la table des matières }}$

S’il s’opère une fermeture à toute intrusion de la parenté, il en va un peu de même en ce qui a trait à la «sphère publique» en général. Plusieurs des jeunes interrogés expriment en effet le sentiment que non seulement le milieu familial a tout ce qu'il faut pour répondre aux besoins de l'enfant mais qu'en plus, il doit le faire et non s'en remettre à d'autres -personnes ou institutions :

Pour moi, c'est les parents qui sont chargés de l'éducation d'un enfant. Moi j'ai l'intention d'avoir une relation avec mon enfant, je ne veux pas le laisser élever par d'autres. (Richard, 24 ans, un enfant de 2 ans, DEC).

Chez plusieurs jeunes pères, la petite enfance est envisagée comme la seule période de la vie où les parents ont le plein contrôle sur les valeurs inculquées, sur les règles, les principes de vie. Cette exclusivité accroîtrait les chances du bon développement de l'enfant, de sa «bonne réussite» sociale et de l'adoption «pour la vie» des valeurs véhiculées par le milieu familial. L'entrée à l'école est alors appréhendée comme la fin du plein contrôle du milieu familial, comme la fin de la petite enfance, comme l'entrée dans le monde social. Il en va de même pour la garderie, à laquelle ils doivent pour la plupart recourir malgré tout -marché du travail ou études oblige-. À cet égard, certains jeunes pères expriment haut et fort la volonté de choisir celle-ci et, plus tard, l'école, selon des critères internes à la vision familiale. En effet, selon eux, il doit y avoir correspondance et prolongement des intentions du processus de socialisation intra-familial à l'extérieur du cercle d'influence :

Ma conjointe passe du temps avec à la garderie avant de la ramener, c'est quelque chose qu'on trouve important aussi là, que [notre enfant] ne considère pas la garderie juste comme étant un endroit où on la dump là, mais comme étant un endroit qui fait partie de notre quotidien à nous autres aussi et du sien. (Mathieu, 24 ans, baccalauréat, un enfant de trois ans) 
La garderie et l'école semblent appréhendées dans ce cas-ci comme une extension du cercle familial. Leur philosophie doit concorder avec celle du système familial. On retrouve donc une forme d'appropriation, par les parents, de ce milieu qui se situe hors des limites du foyer familial et ce, pour le bien-être de l'enfant. Cette sphère publique est incorporée à leur quotidien. On peut à cet égard faire l'hypothèse que lorsqu'une institution (père ou famille) se trouve en profonde transformation, elle est amenée à renouer avec ses «anciennes» fonctions (l'éducation dans ce cas-ci). On peut ainsi mesurer la place occupée par la sphère privée des rapports sociaux lorsque l'on envisage ses intrusions (possibles ou effectives) à l'intérieur de l'espace public. Les propos de Roussel (1975 : 375) sur le devenir de l'individu, s'appliquent bien à cet égard à la paternité : «Plus la société deviendra anonyme, plus l'individu cherchera dans la relation affective la reconnaissance de son identité et la résolution de ses insatisfactions; plus il refusera aussi dans sa vie privée toute norme étrangère à la subjectivité».

\section{Les valeurs privilégiées dans la définition de la paternité}

$\underline{\text { Retour à la table des matières }}$

Si les jeunes pères ont parlé de la famille, notamment de leur idéal de famille quand ils décrivaient leur nouvelle vie, ils ont surtout tenu à parler de leur expérience de père de leur relation au quotidien avec l'enfant, qui est avant tout d'ordre relationnel, à forte dimension affective, comme on va le voir maintenant.

\section{Un rôle paternel basé sur la présence}

\section{$\underline{\text { Retour à la table des matières }}$}

La valeur qui ressort de façon prépondérante eu égard au rôle paternel est la présence auprès de l'enfant, présence dans et par le jeu mais aussi dans les «soins de base», bref, présence au quotidien: 
Un père, à mon avis, c'est quelqu'un avec qui tu peux partager des affaires. C'est quelqu'un qui est présent, qui vit avec l'enfant, qui est là pour l'épauler, qui joue beaucoup avec. Un jeu vidéo, ça n'achète pas la présence d'un père, c'est ça que je pense. (Alexis, 19 ans, un enfant de 8 mois, secondaire 4).

Ça demande énormément d'amour et d'attention. Concrètement, c'est tout le temps être là, tout le temps avoir l'attention, pis du contact. Si tu ne le vois pas de la semaine, je ne crois pas à ça. Je pense qu'il faut que ton enfant apprenne à te connaître autant que toi t'apprends à la connaître, puis ça, bien ça se fait à tous les jours, pus ça se fait au quotidien, tout le temps rester disponible, garder une certaine présence tout le temps. (Charles, 19 ans, un enfant d'un an, DES, travailleur dans une usine de plastique)

La présence à l'enfant, c'est aussi une proximité relationnelle, affective, qui s’exprime parfois autour de simples moments de tendresse. Pour ces pères, il s’agit non seulement de ressentir cet amour, qui est un peu le cœur de la relation, mais aussi de le démontrer en étant tout simplement là, présent :

Quand elle, elle a le goût d'écouter la télé, ce n’est pas parce qu'elle a le goût d'écouter la télé que je vais faire du ménage pendant ce temps là. Je peux aller m'écraser avec elle, m'asseoir avec pourquoi pas. L’émission est plate ce n'est pas grave, je me ferme les yeux, elle s'accote sur moi, je relaxe puis je suis avec. (Mathieu, 24 ans, baccalauréat, un enfant de trois ans, travailleur)

La relation avec l'enfant repose en partie sur l'affect ressenti et l'espace partagé tout en se construisant à partir d'un rapport corporel avec l'enfant. Un bon père, pour eux, c'est celui qui est disponible, qui est à l'écoute, et c'est aussi celui qui est proche de ses enfants à la fois physiquement et psychologiquement. Les qualités que doit posséder un père «idéal» et qui représentent autant de valeurs au sens premier de «manière d'être ou d'agir qu'une personne ou une collectivité reconnaissent comme idéale» (Rocher 1969: 56) sont donc la disponibilité, l'attention, la présence continue et active («être vraiment là») et la proximité (être proche dans tous les sens: contacts physiques, intimité et authenticité de l'échange, établissement d'un rapport de confiance, etc.). 


\section{Une relation père/enfant sous le signe de la démocratie familiale}

$\underline{\text { Retour à la table des matières }}$

Être père, c'est aussi, pour beaucoup de jeunes interrogés, être un guide, un «formateur» auprès de l'enfant. Il ne s'agit pas d'interdire, mais d'expliquer le plus honnêtement possible :

Le rôle d'un parent, c'est de doter l'enfant, pas de lui imposer, mais de lui inculquer une certaine valeur, puis de cultiver son imagination (Richard, 24 ans, un enfant de 2 ans, DEC, étudiant à temps plein)

Le père se voit comme un phare pour l'enfant, indiquant les chemins possibles, sans pour autant lui tracer la voie. Cette vision est d'ailleurs congruente avec les qualités mêmes qui sont, selon eux, à développer chez l'enfant, pour son évolution progressive en société, soit la confiance, l’autonomie, la capacité de choisir. Autrement dit, pour les jeunes pères, l'important est que l'enfant apprenne à opérer des choix pour ensuite être en mesure de se mouvoir confortablement dans un monde marqué par l'autonomie et l'individualisation des parcours de vie. Et la tâche du père est celle, justement, de l'accompagner dans ces sélections, d'être présent lors de dilemmes décisionnels. Le père apparaît donc comme un référent pour son enfant, un phare se voulant visible pour l'ensemble de sa vie, sans pour autant orienter son trajet.

Moi je pense qu'un père c'est quelqu'un qui est là, qui les aide à leur développement (...) puis en leur offrant le plus de possibilités pour qu’ils puissent connaître la vie aussi de leur propre vision, qu’ils puissent se faire eux autres même leur propre image du monde qui les entoure. Leur donner finalement les meilleurs outils pour qu'ils puissent se développer. (Benoît, 24 ans, deux enfants de 2 ans et 2 mois, certificat universitaire, travailleur en garderie et étudiant)

À cette vison du père comme guide correspond un malaise marqué avec la dimension hiérarchique du rapport à l'enfant et surtout avec l’autorité. On sent 
ainsi, dans beaucoup d'entrevues, la volonté de se distancer d’un rapport basé sur l'autorité au profit d'une relation marquée par le dialogue, la communication. Quand il s'agit d'user de l'autorité, c’est de façon dosée et tout en favorisant au préalable l'usage de la parole entre les parties en litige. La discipline pure et dure n’apparaît pas être constitutive de leur pratique paternelle : elle semble plutôt être utilisée de façon occasionnelle et doit toujours être justifiable. Leur façon d'exercer l'autorité est plus «pédagogique» qu'exclusivement punitive, en partie à cause de leur représentation de l'enfance et parce qu'ils sont en complicité quotidienne avec leur enfant. Une relation basée sur la communication vient également conforter un espoir que plusieurs ont exprimé, soit celui de préserver à long terme la proximité émotionnelle avec l'enfant. À cet égard, certains disent vouloir se départir de façons de faire acquises dans leur premier cercle de socialisation, soit leur famille d'origine. Plus spécifiquement, leur propre père est parfois considéré comme un contre modèle et ce, non pas pour l'ensemble de leur pratique paternelle, mais plutôt en ce qui concerne justement la gestion de l'autorité.

\section{Conclusion}

$\underline{\text { Retour à la table des matières }}$

Les jeunes pères semblent donc avoir fait leurs les traits de la famille contemporaine autrement nommée «famille relationnelle» (de Singly 1993) au sein de laquelle prédomine la dimension affective, la place centrale accordée à l’enfant la le souci de se mettre à son niveau, d'être attentif à ce qu'il dit. Ils sont les représentants, pourrait-on dire, de «la nouvelle paternité» à condition de voir dans celle-ci non pas une «mode» mais bien le résultat des transformations dans les valeurs autour de la famille, de la parentalité, de la masculinité. Cette nouvelle paternité a des racines qui plongent à la fois dans les représentations de l'enfant qui ont émergé à partir de l'âge classique, dans les principes anti-autoritaristes et égalitaristes de la contre-culture, dans les nouvelles modalités des rapports hommesfemmes dans la sphère domestique (revendication de l'égalité, partage des tâches), et dans les transformations du masculin (rapport des hommes à leurs émo-

tions et à l'intime). Tout le rapport à l'enfant est marqué chez eux par la centralité de celui-ci dans leur vie, par un souci de respecter certaines règles du jeu édictées 
par lui et d'être attentif à ce qu'il dit. L'enfant, pour ces pères, est donc appréhendé pour lui-même, pour ce qu’il est, comme une «personne» en devenir, dont il faut développer et non brimer le potentiel. C’est peut-être bien cette réceptivité et ce mode de disponibilité paternelle à l'enfant, éléments très frappant dans toutes les entrevues, qui exprimerait le mieux la nouvelle paternité chez les jeunes pères d'aujourd'hui.

\section{Bibliographie}

$\underline{\text { Retour à la table des matières }}$

Arama, D., C. Bouchard (1996) Recension des projets d'intervention ayant trait à la paternité dans la grande région de Montréal, Les cahiers d'analyse du GRAVE, Vol. 3, No. 1, UQAM, 1996.

Bawin-Legros, B., M. Sommer (1987) «Famille/Familles: difficiles et mouvantes typologies, Revue Internationale d'action communautaire, Vol. 18/58, p. 47-55.

Broderick, C. (1977) «Fathers», Family Coordinator, Vol. 26, N. 3, pp. 269275.

Castelain-Meunier, C. (2002). La place des hommes et les métamorphoses de la famille, Paris, PUF.

Cordell, A.S., R.D. Parke, D.B. Sawin (1980), Fathers' Views on Fatherhood with Special Reference to Infancy, Family Relations, vol. 29, no. 3: 331-338.

Dandurand, R.B. (1995). «Jeunes adultes et vie familiale», Acte de colloque du 62e congrès de l'ACFAS, Conseil permanent de la jeunesse.

Delaisi de Parseval, G. (1982). «Les «nouveaux pères» en France», Les pères aujourd'hui, Conseil Supérieur de l'information sexuelle, de la régulation des naissance et de l'éducation familiale, Paris, Éditions de l'INED, p. 13-16. 
Delumeau, J., D. Roche (1990), Histoire des pères et de la paternité, Paris, Larousse.

De Singly, F. (1993), Sociologie de la famille contemporaine, Paris, Nathan.

Dienhart, A.(1998). Reshaping Fatherhood. The Social Construction of Shared Parenting, Thousand Oaks, Sage.

Dulac, G. (1993), La paternité: les transformations sociales récentes, Québec, Conseil de la famille.

Dycke, N., J-F. Saucier (1999). Cultures et paternités, Montréal, Saint-Martin.

Erickson, R., V. Gecas (1991), «Social Class and Fatherhood», in F. Bozett et S. Hanson, Fatherhood and Families in Cultural Contexts, New York, Springer, p.114-137

Ferrand, M. (1981). «La paternité dite par les hommes », Le Groupe Familial, no 92, p. 60-64.

Fournier, F., A. Quéniart (1994). Les formes contemporaines du rapport à la parentalité chez les pères québécois: essai de typologie sociologique, rapport de recherche, Laboratoire de recherche en écologie humaine et sociale, UQAM.

Gauthier, Madeleine, Bernier, Léon, et al. (1997). Les 15-19 ans : quel présent ? vers quel avenir ?, Sainte-Foy, Institut québécois de recherche sur la culture.

Erickson, R., V. Gecas (1991), «Social Class and Fatherhood», in F. Bozett et S. Hanson, Fatherhood and Families in Cultural Contexts, New York, Springer, p.114-137

Hurstel, F. (1987), La fonction paternelle aujourd'hui : problèmes de théorie et questions d'actualité, Enfance, op.cit.: 163-179. 
Iishii-Kuntz, M. (1994), «Paternal Involvement and Perception Toward fathers' Role: A Comparison Between Japan and the United States», Journal of Family Issues, Vol.15, No.1, p. 30-48.

Kerr, D., D. Larivée, P. Greenhalgh (1994). Les enfants et les jeunes : un aperçu, Ottawa, Statistique Canada, Prentice-Hall, Canada.

Lamb, M.E. et al. (1987), The Father's Role: Cross Cultural Perspectives, Hillsdale (New Jersey), L. Erlbaum.

Lacharité, C. (2001). «Comprendre les pères vivant dans des environments défavorisés», dans Forget, G. Présence de pères, Montréal, Direction de la santé publique, p 57-61.

Laperrière, A. (1998). «La théorisation ancrée (grounded theory) : démarche analytique et comparaison avec d'autres approches apparentées», Partie4, chapitre 2, dans Poupart, J. et al., La recherche qualitative. Tome 1 : enjeux épistémologiques et méthodologiques, p. 309-340.

Lévesque, P-A., M. Perrault, C. Goulet (1997), «La paternité en milieu défavorisé : le point de vue d’intervenants sociaux». Père à part entière, sous la direction de J. Broué et G. Rondeau, Montréal, Éditions Saint-Martin, p. 91-112.

Martin, C. «Solidarités familiales: l’illusion du renouevau», Familles. Permanence et métamorphoses, Paris, Ed. Sciences Humaines, 2002.

Ménahem, G. (1979). «Les mutations de la famille et les modes de reproduction de la force de travail», L’homme et la société, p.12-23.

Paillé, P. (1994). «L’analyse par théorisation ancrée», Cahiers de recherche sociologiques, No. 23, p. 141-181.

Palm, G.F., R. Palkovitz (1988). «The Challenge of Working with New Fathers: Implications for Support Providers», Marriage and Family Review, vol. 12, n 34, p. 357-376. 
Pleck, J.H. (1987). «American Fathering in Historical Perspective», Changing Men: New Directions in Research on Men and Masculinity, Micheal S. KIMMEL editor, Newbury Park, CA, Sage, p. 83-97.

Quéniart, A., É. Lachance (1995). Les études sociologiques sur la paternité: transformations socio-historiques, représentation et pratiques, document de recherche, département de sociologie et Laboratoire de recherche en écologie humaine et sociale (LAREHS), UQAM.

Quéniart, A. (2000), «Qui sont les pères aujourd'hui?», Interface, Vol. 21, No.1, p. 35-41.

Quéniart, A. (2002a). «La paternité sous observation: des changements, des résistances mais aussi des incertitudes», dans Descarries, F. et C.Corbeil (dir.). Espaces et temps de la maternité, Montréal, remue-ménage, p. 501-522.

Quéniart, A. (2002b). «Place et sens de la paternité dans les projets de vie des jeunes pères», dans Pronovost, G. (dir.). Comprendre la famille, Montréal, PUQ, p. 55-76.

Robinson, B.E., R.L. Barret (1986). The Developing Father: Emerging Roles in Contemporary Society, New York, Guilford.

Rocher, G. (1969). Introduction à al sociologie générale. Tome 1. Regards sur la réalité, Montréal, $\mathrm{HMH}$.

Rotundo A. E. (1985). «American Fatherhood», American Behavioral Scientist, p. 7-25.

Roussel. L. (1980). «Mariages et divorces. Contribution à une analyse systémique des mariages matrimoniaux», Population, 6,

Roussel, L. (1975), Le mariage dans la société française, Paris, PUF. 
Secrétariat à la Jeunesse (1992). La Jeunesse québécoise : faits et chiffres (1529 ans), Québec, Ministère du Conseil exécutif.

Russel, G. (1982). «Highly Participant Australian Fathers: Some Preliminary Findings », Merril Palmer Quartely, vol. 28, n 1, p. 137-156.

Secrétariat à la Jeunesse, La Jeunesse québécoise : faits et chiffres (15-29 ans), Québec, Ministère du Conseil exécutif, 1992.

Sennett, R. (1979), Les tyrannies de l'intimité, Paris, Seuil.

Turcotte, G. (1994), L'implication paternelle: déterminants et modèles d'intervention, Les cahiers d'analyse du GRAVE, Laboratoire de recherche en écologie humaine et sociale, Montréal.

Verheyen, C. (1987). «Mother Knows Best: For Him the Play, For Her the Rest», dans Unravelling Fatherhood, sous la dir. de T. Knijn et A-C. Mudler, Dordrecht, Foris Publications, p. 37-47.

\section{Fin du texte}

